\title{
Modeling and implementation of smart home and self-control window using FPGA and Petri Net.
}

\author{
AJAO, L.A., AGAJO, J., UMAR, B.U., AGBOADE, T.T. and ADEGBOYE, \\ M.A.
}

(C) 2021 IEEE. Personal use of this material is permitted. Permission from IEEE must be obtained for all other uses, in any current or future media, including reprinting/republishing this material for advertising or promotional purposes, creating new collective works, for resale or redistribution to servers or lists, or reuse of any copyrighted component of this work in other works. 


\section{Modeling and Implementation of Smart Home and Self- control Window using FPGA and Petri Net}

\author{
Lukman Adewale Ajao \\ James Agajo \\ Buhari Ugbede Umar \\ Tope Tobi Agboade \\ Department of Computer Engineering \\ Federal University of Technology \\ Minna, Nigeria \\ ajao.wale@futminna.edu.ng, \\ ORCID: 0000-0003-1255-752X
}

\author{
Mutiu Adesina Adegboye \\ Department of Computer Engineering \\ Federal University Oye-Ekiti \\ Ekiti, Nigeria \\ mutiu.adegboye@fuoye.edu.ng, \\ ORCID: 0000-0003-3053-986X
}

\begin{abstract}
The function of the window is to provide comfort for the householders by regulating the indoor environment. However, most of the residence windows are still controlled manually. Although, a quite number of automated windows based on the Internet of Things (IoT) has been proposed in the literature, yet a smart windows control with multi-objectives functions related to the environmental factors remain open gap. This paper aims to contribute to this area by developing a cyberphysical system (CPS) for smart room and windows automation control (SRWAC) using set of rules generated from the Petri Net simulator for determining the system response to the inputs data sensed from the indoor and outdoor sensing units (temperature, dust, rain, and carbon monoxide sensors) in coding Atmega 328 controller. We also simulate the window controller using Field Programmable Gate Array (FPGA) in Xilinx ISE. The results of the system tested show that automated response of the indoor and outdoor conditions can be achieved spontaneously.
\end{abstract}

Index Terms - Cyber-physical system, Field programmable gate array, Home automation system, Internet of Things, Smart room.

\section{INTRODUCTION}

The building is usually erected to provide shelter and as well enable man to dwell on the earth. Any opening in a wall, door, or roof that allows passage of air, light, and sometimes sounds is known as window [1]. Weather condition is dynamic, and changes vary from time to time across the different geographical regions. These changes always influence the indoor environments, while maintenance of indoor ventilation plays an important role in human health. Opening windows is also encouraged in the medical park, hotel, laboratory, and some industrial application areas [1]. Whereas the ordinary window that requires physical contact often brings inconvenience to aging and people with disabilities. The statistic shows that the number of people in the world aged 60 years or over is expected to be more than double between 2015 and 2050, reaching nearly 2.1 billion [2].

Advancement in science and technology has made automation of the window possible, and it has become part of our daily lives. This has contributed immensely to the security and living standard of the human being through the application of the Internet of Things (IoT), Industrial IoT (IIoT), and Cloud of Things (CoT) computations [3]. Research on IoTs has progressed in recent times [4]. Its emergence has added more dimensions to the advancement of information and communication technologies [5]. IoT enables the users to achieve home automation systems, which help to save time, attaining energy efficiency, and maintain a certain level of comfort. The computer-based algorithms that integrated with the internet and users are referred to as Cyber-Physical Systems.

Windows is an essential component of every structure, whether residential or office, and it requires a certain level of intelligence before it can adjust itself based on environmental conditions. The effectiveness of smart home automation devices depends on embedded sensors, system control units, and wireless technology, which brings convenience to the home occupant. This smart appliance includes refrigerators, doors, lighting, thermostat, and many others [2]. The performance of the IoT ecosystem is based on the collections of environmental data. Such information is transforming to other devices for controlling and activates the actuators through wireless protocols (Wi-Fi, Bluetooth, ZigBee, ZWave, and so on) and the internet.

The smart home automation model aims at logically connecting all the appliances or build a centralized home entertainment for seamlessly control, monitoring, and security purposes at our fingertip [6], [7]. This smart activity could be possible through IoT or cloud computing using internet protocols. A machine learning technology (MLT) can also be adopted in the IoT to condition a controller using IF This Then 
That (IF3Ts or IFTTT) method for the efficient control and better performances of the systems [8]-10]. This approach will enable the embedded system experts or developers to create a cascading effect of actions to satisfied smart system design, achieve the optimum use of home automation systems, and managing home energy simultaneously for a comfortable lifestyle.

In this paper, an attempt has been made to design a smart room and automated window control, which can automatically open and close based on the changes in indoor and outdoor weather conditions such as temperature, dust, rain, and carbon monoxide. The contributions of this paper include window control strategies modeled using Field Programmable Gate Array. The Atmega 328 coding was using a set of rules generated from the Petri Net simulator. A laboratory prototype was built and tested using different predefined weather conditions. This system would benefit aging, disabled, and people who have less time to spare on opening and closing windows. The rest of article is arranged as follows. Section II presented literature review, section III discussed cyberphysical implementation of smart room and window automation control. Section IV presented modeling of cyberphysical system of smart room and window automation control using petri net. Section V presented results and discusson, while section VI conclude the research findings.

\section{LITERATURE REVIEW}

The motivation behind the implementation of smart home and the automatic window is to provide householders safety, independent leaving, security, and comfort. In this regard, a variety of smart home related research has been proposed in the literature. A recent study by Liu et al. [11] designed an intelligent window system based on the platform of Newland Group. In this work, the designed system was divided into two parts, namely host monitoring and remote monitoring platforms. In the host monitoring platform, different sensors include rain, noise, burglary, and carbon monoxide concentration sensors, were connected for weather conditions monitoring and control. The remote monitoring platform was designed for the users to obtain real-time data, adjust open window percentage, and update the occupant by text message.

Liqing et al. [12] developed a control switch for open and closed windows automatically based on the indoor and outdoor environment changes. The indoor combustible gas was monitor using the MQ-2 sensor, while the infrared probe was utilized for the outdoor. Also, monitoring in this work includes temperature, humidity, and indoor light intensity. Stepper motor was employed to change the status of the window based on the information collected from the sensors.

An intelligent window based on the embedded fuzzy logic system was proposed by Tian et al. [13]. A fuzzy inference modeling was established with the two input variables (temperature and wind speed). The ARM chip S3C2410 was used as the main controller, while the step-motor was utilized for driving the window. The system was designed such that different positions of the window can be tracking using Hall sensor. The system performance shows that auto and remote control of the window is possible with the use of fuzzy inference control.
Kan et al. [14], developed a model to create a window automation system for health and environmental monitoring with various wireless sensor network devices. The control operation of the designed system was performed using a micro control unit, while the radio frequency and wireless sensor network antenna were utilized for receiving the command from the embedded. Other related studies include smart home security, energy efficiency management, and so on [15].

\section{PRoposed SMART RoOM AND Window Automation CONTROL USING MICROCONTROLLER}

The adoption of the cyber-physical smart system (CPSS) for room and window automation involved the integration of several hardware components and software programming. The hardware parts of CPSS include the embedded Atmega 328 controller chip, wireless sensors (such as MQ-9 gas sensor, MLX90614 temperature sensor, a dust sensor, and water sensor), servo motor, and IEEE $802.11 \mathrm{~b} / \mathrm{g} / \mathrm{n}$ wireless communication protocol. All these peripherals are the interface with an intelligent controller chip for the processing of environmental data acquisition and give output response through the implemented actuator (servo motor), as illustrated in Fig. 1.

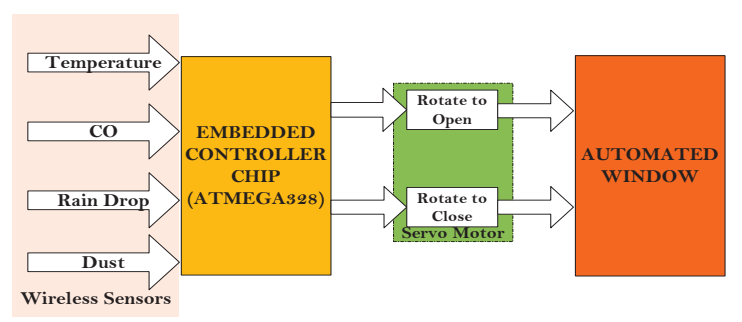

Fig. 1. System block diagram

The smart room and window automated design is divided into two units, which include the indoor system unit and outdoor system unit. Both system units are communicating with each other and through the intelligent chip using a wireless communication protocol (IEEE $802.11 \mathrm{~b} / \mathrm{g} / \mathrm{n}$ ). The software coding technique is in two folds. The first one is embedded codes used for controlling hardware devices and wireless communication systems. This was programmed in the Arduino integrated development environment (AIDE) using C-language. The second part of the software is the coding for developing android mobile apps using Java programming language with the help of android studio IDE for remote monitoring and control.

Also, the cyber-physical system for smart room and window automation is modeling in Petri Net system simulation using the philosophy model approach of dining arrangement. The output response was implemented in Field Programmable Gate Array (FPGA) for industrial application purposes and enhancement in smart technology. The indoor and outdoor system units consist of wireless transmitters to enable communication with the central control unit using a wireless radio frequency of $2.4 \mathrm{G} \mathrm{Hz}$. Both system modules are deployed within the smart room and window environment for the easiest communication to the central control unit.

The indoor system unit (ISU) is mounted within the room for room temperature related to the occupancy. This ISU 
consists of MLX90614 temperature sensor and carbonmonoxide (CO) sensor, in which the circuit implementation is simulated in Proteus Virtual Simulation Modelling (PVSM) as illustrated in Fig. 2. The MLX90614 temperature sensor is a sensitive infrared (IR) contactless digital thermometer with high accuracy that can be used for the measurement of temperature between $-70^{\circ} \mathrm{C}$ to $382.2^{\circ} \mathrm{C}$. This sensor utilizes the IR rays to measure the human temperature inside the room without any physical contact and communicate to the controller through the $\mathrm{I} 2 \mathrm{C}$ protocol for the response.

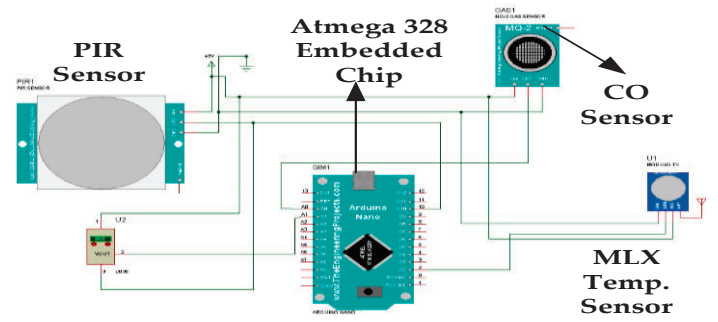

Fig. 2. Simulated circuit diagram of the indoor unit

The Carbon monoxide (CO) sensor is deployed to sense the room environment for unburned fuel detection that can cause or produce gas from a smart device burning such as clothes dryers, water heaters, fire-burning fireplace, ovens or gasoline and others related to incompletely burning fuels. The MLX90614 temperature sensor is used for sensing the room temperature environment. The Passive Infrared Sensor (PIR) is used in motion or movement detection by measuring the infrared light radiating from objects in its Field of View (FoV). This PIR sensor is working based on the principle of Frieden's model assumptions that have uniform temperature distribution, and it can be expressed as given in Equation 1.

$I=\frac{2 \alpha \sigma \beta}{\pi H c} B T_{a}^{2} \frac{\left(T_{b}-T_{a}\right)}{D^{2}}$

where $I$ is the current generated by the sensor, $\alpha$ is the pyroelectric sensor coefficient, $\sigma$ is the Stefan-Boltzmann constant, $\beta$ is the lens efficiency, $H$ is thickness, $c$ the specific heat of the sensing material, $B$ is the effective surface area of the subject, $T_{a}$ is the sensor temperature, $T_{b}$ is the surface temperature of the object, $D$ is the distance between the object and the sensor.

The outdoor system unit (OSU) is mounted at the window side of the room, and it consists of a dust sensor and a water sensor. Both sensors are configured to sense the drop of water and dust for the immediate controlling of the window through the actuator device (servo motor). The outdoor environmental data is acquired through OSU and sent the parameters to the central control unit via the wireless device, as given in Fig. 3.

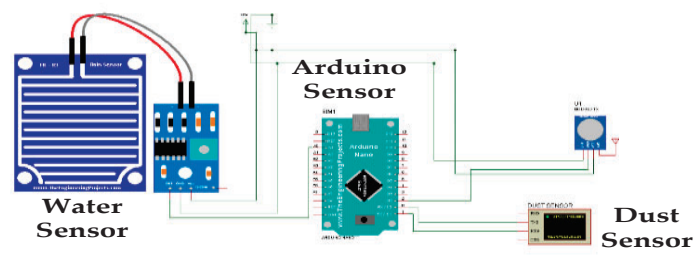

Fig. 3. Circuit diagram of the outdoor unit
The water sensor element is integrated into the OSU for water drop detection or sensing of rainfall. The advantage of this device is efficient for water level detection, volume, and plant irrigation. The water sensor consists of three pins (including S, Vout, and Ground). The pin S is connected to the digital input/output of Arduino for the data communication; the Vout is connected to the $5 \mathrm{~V}$ power supply and Ground (GND) to the Arduino board, respectively. The GP2y101AU0F optical air quality sensor is used for dust particles detection during rain clouds or windstorms through an infrared emitting diode and phototransistor integrated into the device.

\section{SMART ROOM AND Window Automation Modeling USING PETRI NET AND FPGA}

The smart room and window automation are modeled in Petri Net simulation software to validate the cyber-physical implementation of smart technology in the home automation system, as presented in section III. The philosophy idea from this modeling is implemented in FPGA to replicate the same functions of home automation control. This design technology approach is incredible and perfect in the technical application of embedded system development, mechatronics system, and robotics. It proves to be efficient, reliable, and seamless for the future of industrial applications towards the internet of things (IoT). It is well available and interactive for learning purposes, laboratory experiment simulations, and use for experts and researchers in computational intelligence and machine learning application.

This modeling assumed to be a real-life scenario for a smart home automation system with the implementation of smart appliances. The components used in the modeling include places $\left(\mathrm{P}_{1}, \mathrm{P}_{2}, \mathrm{P}_{3} \ldots \mathrm{P}_{\mathrm{n}}\right)$, Transitions $\left(\mathrm{T}_{1}, \mathrm{~T}_{2}, \mathrm{~T}_{3}, \ldots \mathrm{T}_{\mathrm{n}}\right)$, Token which can either be 0 or 1 for the active and inactive functions of the wireless sensors and the connecting wires which can be single or bi-directions. The philosopher prototypical of using Petri Nets (PN) for this smart technology development is the basic model for parallel and distributed systems, which can be described as (P, T, I, O, Mo). To determine the design structure matrix called marking $\left(\mathrm{M}_{\mathrm{o}}\right)$ is expressed as given in "(7)".

$$
\begin{aligned}
& P_{N}=\left(\alpha, \beta, \gamma, \vartheta, M_{0}\right) \\
& \alpha=\alpha_{1}, \alpha_{2}, \alpha_{3}, \ldots \alpha_{n} \\
& \beta=\beta_{1}, \beta_{2}, \beta_{3}, \ldots \beta_{n} \\
& \zeta=\left(\begin{array}{lll}
\alpha_{1} \rightarrow \beta_{1} & \alpha_{2} \rightarrow \beta_{1} & \alpha_{n} \rightarrow \beta_{1} \\
\alpha_{1} \rightarrow \beta_{2} & \alpha_{2} \rightarrow \beta_{2} & \alpha_{n} \rightarrow \beta_{n} \\
\alpha_{1} \rightarrow \beta_{n} & \alpha_{2} \rightarrow \beta_{n} & \alpha_{n} \rightarrow \beta_{n}
\end{array}\right)_{n} \\
& \vartheta=\left(\begin{array}{lll}
\beta_{1} \rightarrow \alpha_{1} & \beta_{1} \rightarrow \alpha_{2} & \beta_{1} \rightarrow \alpha_{n} \\
\beta_{2} \rightarrow \alpha_{1} & \beta_{2} \rightarrow \alpha_{2} & \beta_{n} \rightarrow \alpha_{n} \\
\beta_{n} \rightarrow \alpha_{1} & \beta_{n} \rightarrow \alpha_{2} & \beta_{n} \rightarrow \alpha_{n}
\end{array}\right)_{n}
\end{aligned}
$$

where, $P_{N}$ is the Petri Net (Bipartite graph), $\alpha$ is the set of places in the graph, $\beta$ is a set of transitions in the graph, $\zeta$ is a 
function of input in the model, $\vartheta$ is a function of output in the model and $M_{o}$ is called marking, is an assignment of tokens to the places in a bipartite graph (Petri nets).

The PN has a different degree of liveness $\left(L_{1}-L_{4}\right)$, and a $P_{N}$ $\left(N, M_{o}\right)$ is called $\mathrm{L}_{\mathrm{k}}$-live if and only if all transitions are $\mathrm{L}_{\mathrm{k}}-$ live.

i. If a transition is dead ( $\mathrm{L}_{\mathrm{k}}$-live), the transition can never be fired. That is, it is not in any firing sequence of $L(N, M o)$. No activation or placing of the token inside a place.

ii. If a transition is potentially fireable ( $\mathrm{L}_{1}$-live), it can fire, if and only if it may be firing in some sequence of $L(N$, $\left.M_{o}\right)$.

iii. Fire arbitrarily ( $\mathrm{L}_{2}$-live), if, for every positive integer $\mathrm{k}$, it occurs at least $k$ times in some firing sequence in $L(N$, $M_{o}$ ).

iv. Infinitely Fireable ( $\mathrm{L}_{3}$-live), if there is some fixed (necessarily infinite) firing sequence for every positive integer $k$, the transition $\mathrm{L}_{3}$ occurs at least k-times.

v. Always fireable ( $\mathrm{L}_{4}$-live), It is $\mathrm{L}_{1}$-live in every reachable marking in $R\left(N, M_{o}\right)$.

The first scenario model of this smart room environment measured the prevalence of temperature and carbon monoxide, and the second scenario considered the incidence of dust particle and water spilled detection for window automation, as illustrated in Fig. 4 and 5.

Scenario 1: IF Temperature is (High), CO is detected (High), AND no Rain (Low), no Dust Detected (Low), THEN the transition (t18, t19 and $\mathrm{t} 24)$ is fireable to activate the servo motor one and automate "OPEN WINDOW."

Scenario 2: IF Temperature is (Low), CO is (Low), AND Water drop detected (High), Dust is detected (High), THEN the transition ( $\mathrm{t} 1, \mathrm{t} 3, \mathrm{t} 4, \mathrm{t} 6, \mathrm{t} 22, \mathrm{t} 23$, and $\mathrm{t} 25)$ is fireable to activate the servo motor 2, and automate "CLOSE WINDOW".

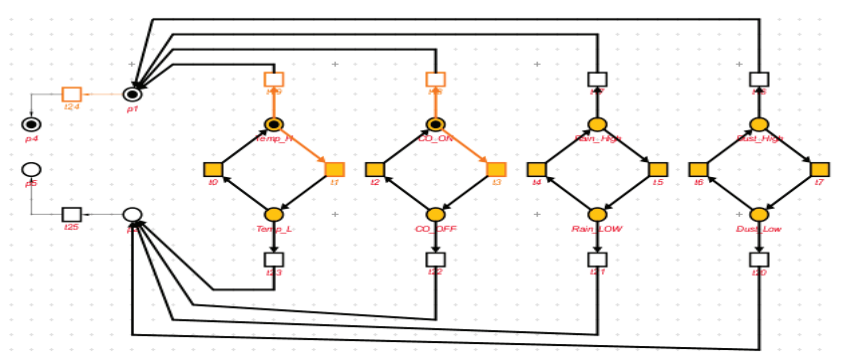

Fig. 4. Temperature and CO scenario model

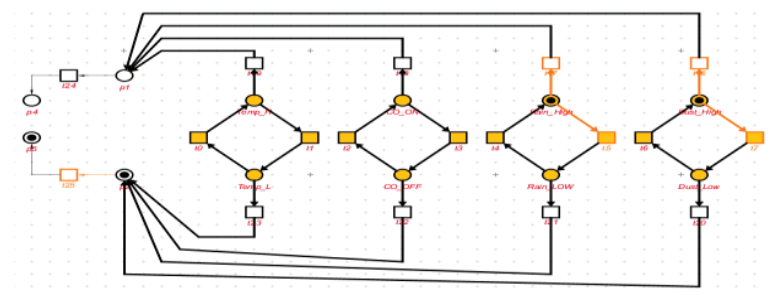

Fig. 5. Rain and dust detection scenario model

\section{RESUlT AND DISCUSSION}

The logical and philosophical reasoning about these scenarios is coding in hardware descriptive language (HDL) and implemented in Field Programmable Gate Array (FPGA). In a concise, the result obtained from using FPGA implementation for smart room and window automation is simple, efficient, and precisely compare to using microcontroller or convention single-on-chip (SoC) device as presented in Fig. 6 and 7. The two input parameters are the "temperature" and "CO", while the output responses are "Open Window" and "Close Window." IF the room temperature is "High" and gas odor detected, the window will be automatically "Open," and IF temperature is "Low" and no gas detection window will remain closed. But, If either one of the conditions is "High" or "Low" the window will be automated to partially opened. The same logic applies to the rain and dust detection during a wind storm or rain-cloud.

Ajao et al. outline the comparative performance between the adoption of FPGA and conventional microcontroller as a benchmark with standard embedded system metrics such as time-to-market, cost, speed, non-recurring expenses (NRE), availability, and failure rate [16].

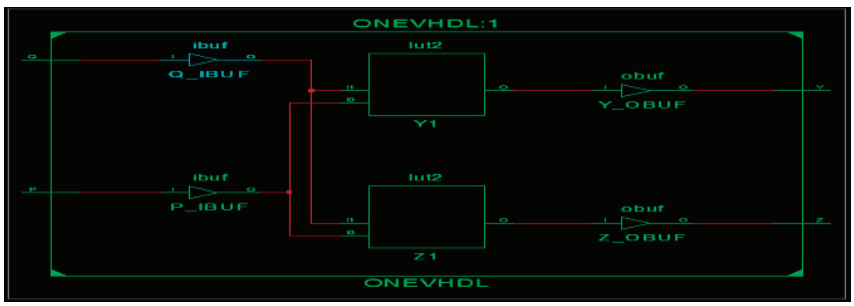

Fig. (6)a. Temperature and CO scenario model

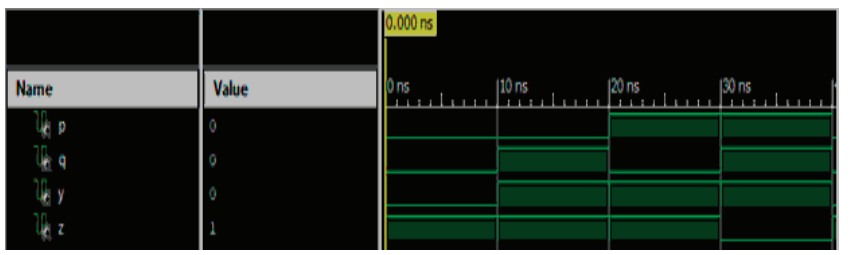

Fig. (6)b. Temperature and $\mathrm{CO}$ detection model

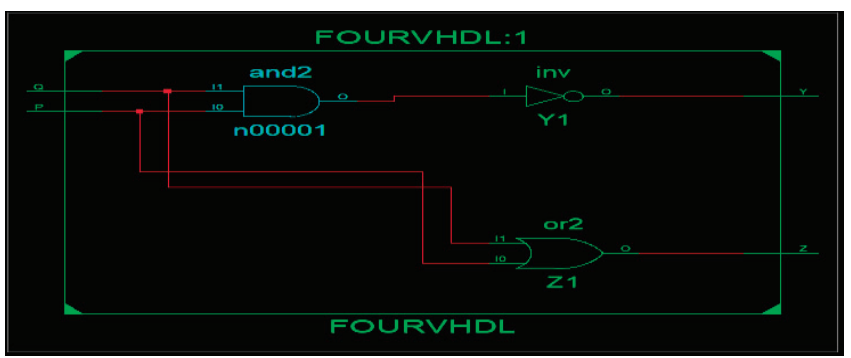

Fig. (7)a. Rain and dust detection model

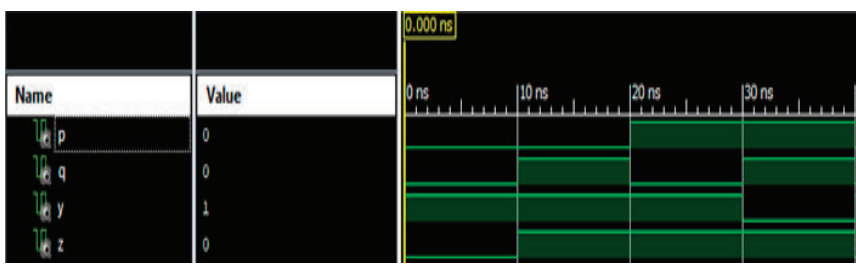

Fig. (7)b. Rain and dust detection 
The laboratory testing and prototypical development of smart home and self-control window automation systems are test-run. The evaluation performance result obtained at an average response time of 3.5 seconds, has an accuracy of $99 \%$ as in Figures 8 and 9, respectively. The response time of a system is a measure of time taken for the input parameters, and time is taken to respond to the mobile apps. While, the accuracy is a measure of input variables, processing, and output response time of the system.

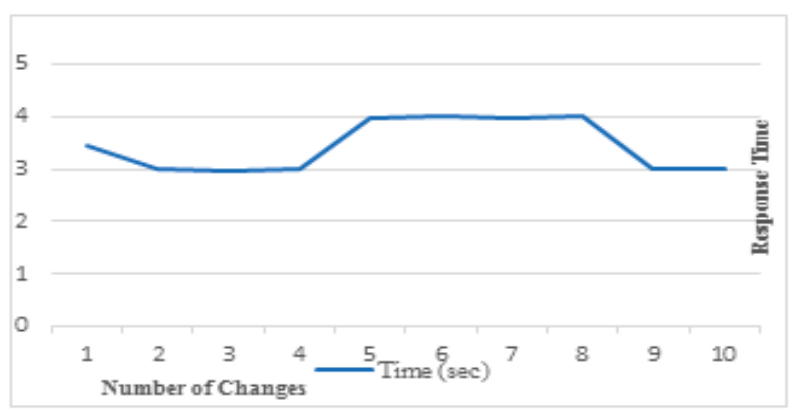

Fig. 8. System response time against the number of trials

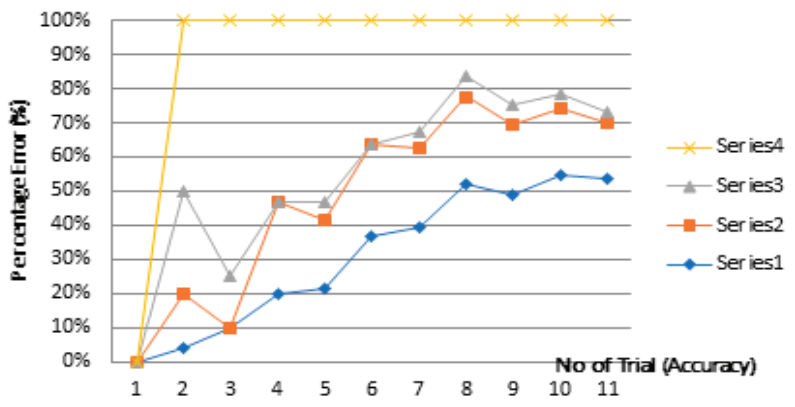

Fig. 9. Accuracy of the system measured against the number of trials

\section{CONCLUSION}

Comfort and safety are crucial issues to be worried about at the aging stage of human lives, and to people with disabilities. So, the smart home automation system is a way out to those challenges of human requirements. Therefore, this research proposed modeling and implementation of smart home and self-control window automation system using ATmega 328 microcontroller and Field Programmable Gate Array (FPGA) in the laboratory prototypical system development. The temperature, gas leakages, burning odor or gaseous emissions, water spilled, and sound storm or dust are considered as the input parameters in the modeling. The Petri Net (a bipartite graph) was adopted in modeling and simulation of this cyber-physical smart home automation and self-control window system. The system operates perfectly as the evaluation result obtained at an average response time of 3.5 seconds has an accuracy of $99 \%$. This work complements the human efforts in response to the home environmental factors, and it would benefit aging, disabled, and people who have less time to spare on opening and closing windows.

\section{REFERENCES}

[1] A. M. Zungeru, J. M. Chuma, M. Mangwala, and L. R. Tlhabiwe, "Design and simulation of a weather activated window system," International Journal of Applied Engineering Research (IJAER), 12(20), 10189-10196, 2017

[2] J. M., Wang, M. T., Yang, and P. L. Chen, "Design and implementation of an intelligent window system using smart handheld device and fuzzy microcontroller," Sensors, 17(4), 1-14, 2017.

[3] M. Al-Kuwari, A. Ramadan, Y. Ismael, L., Gastli, A. Al-Sughair, and M. Benammar, "Smart-home automation using IoT-based sensing and monitoring platform", In 2018 IEEE 12th International Conference on Compatibility, Power Electronics and Power Engineering (CPEPOWERENG 2018, 1-6, 2018.

[4] M. A. Adegboye, O. M. Olaniyan, O. Okwor, and L. A. Ajao, "Internet of things: a survey of the security challenges and countermeasures," A Journal Publication of the Faculty of Science, Adeleke University Ede, 3(2016), 97-105, 2016.

[5] V. Govindraj, M. Sathiyanarayanan, and B. Abubakar, "Customary homes to smart homes using Internet of Things (IoT) and mobile application," In 2017 International Conference on Smart Technologies for Smart Nation (SmartTechCon), 1059-1063, 2017.

[6] S. Aliyu, A. Yusuf, A. Umar, M. Hafiz, and L. A. Ajao, "Design and development of a low-cost gsm-bluetooth home automation system", International Journal of Artificial Intelligent and Application, 9(8), 4150, 2017.

[7] L. A. Ajao, J. G. Kolo, E. A. Adedokun, O. M. Olaniyi, O. C. Inalegwu, and S. K. Abolade, "Smart door security-based home automation system: An Internet of Things". Science Federatin Journal of Telecommunication, 2(2), 1-9, 2018.

[8] H. Yang, W. Lee, and H. Lee, "IoT smart home adoption: The importance of proper level automation," Journal of Sensors, 2018(1), 1$11,2018$.

[9] M. S. Mahamud, M. S. R. Zishan, S. I. Ahmad, A. R. Rahman, M. Hasan, L. Rahman "Domicile-an IoT based smart home automation system", 2019 international Conference on Robotics, Electrical and Signal Processing Techniques (ICREST), 493-497, 2019.

[10] L. A. Ajao, F. J. Ajao, M. A. Adegboye, and A. A. Ismail, "An embedded fuzzy logic-based application for density traffic control system", International Journal of Artificial Intelligence Research (IJAIR), 2(1), 6-13, 2018.

[11] L. Liu, M. Wei, Y. Zou, W. Guo, J. Wu, and J. Rao, "Design of intelligent window system based on cloud platform," In IOP Conference Series: Materials Science and Engineering, 490(4), 42-46, 2019.

[12] L. Geng, Y. Li, S. Shao, D. Liu, and Q. Zhang, "The Design of the Intelligent Window Control System Based on Wireless," In 2018 10th International Conference on Measuring Technology and Mechatronics Automation (ICMTMA), 294-297, 2018.

[13] J. Tian, M. Gao, and J. Li, "Intelligent window based on embedded system," In Eighth International Conference on Software Engineering, Artificial Intelligence, Networking, and Parallel/Distributed Computing (SNPD 2007), 2(1), 395-399, 2007.

[14] Y. C. Kan, H. C. Lin, and W. P. Sung, "Developing an intelligent control system of automatic window motor with diverse wireless sensor network devices," 39(4), 809-818, 2014.

[15] M. A. Shiru, J. G. Kolo, A. Simon, L. A. Ajao, J. Agajo, and B. K. Nuhu, "Smart home energy management system using least square regression analysis", Saudi Journal of Engineering and Technology (SJEAT), 3(6), 357-367, 2018.

[16] L. A. Ajao, J. Agajo, B. O. Abisoye, A. O. Ajao, M. B. Mua'zu, and A. F. Salami, "Automated multiple water tanks control system using ATMEGA and FPGA technology, IEEE 1st International Conference on Mechatronics, Automation and Cyber-Physical Computer System, 346-353, 2019. 